\title{
Implantação e Avaliação de uma Rede Sem-Fio de Longo Alcance e Baixa Potência para Cidades Inteligentes
}

\author{
Jonas Rossato ${ }^{1}$, Fabio A. Spanhol ${ }^{1}$, Edson T. de Camargo ${ }^{1}$ \\ ${ }^{1}$ Universidade Tecnológica Federal do Paraná (UTFPR) - Câmpus Toledo \\ Rua Cristo Rei, 19 - Vila Becker - CEP 85902-490 - Toledo - PR - Brasil \\ Resumo. Redes LPWAN (Low-Power Wide-Area Network) são um tipo de rede \\ sem-fio projetada para cobrir uma extensa área a uma baixa taxa de trans- \\ ferência de bits. Entre as tecnologias LPWAN, o padrão aberto LoRaWAN \\ destaca-se por operar em uma faixa de frequência aberta, atingir um longo \\ alcance, pouco consumo de energia e baixo custo dos equipamentos. Este ar- \\ tigo descreve a implantação e avaliação de uma rede LoRaWAN para apoiar \\ a construção do conceito de cidades inteligentes no município de Toledo/PR. \\ A avaliação considera a qualidade do sinal em diversos pontos do município e \\ confronta os dados obtidos com um modelo de simulação. Também são apresen- \\ tados o plano para expansão da rede e algumas aplicações em desenvolvimento.
}

\begin{abstract}
Low-Power Wide-Area Network (LPWAN) is a type of wireless network designed to cover a large area at a low bit rate. Among LPWAN technologies, the LoRaWAN open standard stands out for operating in an open frequency range, achieving long-range, low power consumption and low equipment cost. This paper describes the implementation and evaluation of a LoRaWAN network to support the construction of the smart cities concept in Toledo/PR. The evaluation considers the signal quality in several points of the city and compares the obtained data with a simulation model. The plan for network expansion and some applications under development are also presented.
\end{abstract}

\section{Introdução}

O crescente avanço do processo de urbanização converteu as cidades em imensos aglomerados populacionais que enfrentam desafios complexos como a otimização do uso de recursos energéticos, a ocupação racional dos espaços geográficos, a minimização de impactos ambientais, a promoção da mobilidade urbana eficiente e outros. Atualmente, no conceito de cidades inteligentes (smart cities), a combinação de tecnologias de sensoriamento, redes sem-fio de longo alcance e infraestrutura computacional para processamento de grandes volumes de dados heterogêneos possibilitam elaborar soluções inteligentes e escaláveis para lidar com tais desafios.

Em uma cidade inteligente, uma rede de comunicação de dados não é utilizada para conectar exclusivamente pessoas, mas qualquer objeto. O conceito de Internet das Coisas ou IoT (Internet of Things) é empregado para definir objetos conectados a Internet capazes de gerar informação e serem representados no mundo virtual [Bardyn et al., 2016]. Tais objetos possuem características particulares quanto ao uso de uma rede: (i) enviam pequenas quantidades de dados periodicamente; (ii) conectam-se diretamente à Internet ou entre si através de um enlace sem-fio; (iii) são ligados a uma bateria e/ou painel solar; (iv) podem estar espalhados por áreas de difícil acesso; (v) estão fixos ou 
em movimento - em um poste ou em um veículo em movimento, por exemplo. Nesse contexto, destacam-se as redes de baixo consumo de energia e longo alcance, conhecidas como LPWAN (Low Power Wide Area Network) [Centenaro et al., 2016].

Uma rede LPWAN permite enviar apenas alguns Kbps periodicamente, mas pode cobrir áreas de até 50 quilômetros em campo aberto. Também proporciona maior vida útil para as baterias dos dispositivos finais (sensores, atuadores) e ocupa faixas de frequência de bandas não licenciadas [Centenaro et al., 2016]. Entre as tecnologias e protocolos de LPWAN destacam-se a SigFox e a LoRaWAN. Embora possuam várias semelhanças, a principal diferença está no custo do licenciamento de uso. A SigFox atua como uma operadora de telecomunicação convencional, instalando a infraestrutura necessária para operar a rede e tarifando o cliente pelo seu uso. Por outro lado, o padrão aberto LoRaWAN não cobra qualquer taxa pela utilização da rede e estimula o próprio cliente instalar sua infraestrutura de rede. Por isso, há a necessidade de trabalhos tanto práticos quanto teóricos que apresentem as experiências na implantação, avaliação e uso de tais redes.

Dentre os trabalhos que realizam experimentos práticos e simulações para avaliar o sinal da tecnologia Lora no Brasil destacam-se [Ortiz et al., 2018, 2019]. No entanto, conforme apontado por [Oliveira et al., 2018], há poucos estudos brasileiros sobre o padrão e, além disso, o desenvolvimento da tecnologia LoRa em território nacional carece de avanços na área, sobretudo implementações práticas em ambientes reais de monitoramento remoto.

Neste contexto, o presente artigo descreve a implantação e avaliação de uma rede LoRaWAN na UTFPR - câmpus Toledo para dar suporte a implantação do conceito de cidades inteligentes no município. Uma colaboração entre a UTFPR e a Prefeitura Municipal pretende implantar a rede em toda a área urbana, servindo como infraestrutura para aplicações como rastreamento de veículos e monitoramento dos parâmetros da qualidade do ar e água. A UTFPR está localizada em uma região que permite avaliar os sinais da rede tanto na área rural quanto urbana. Assim, foram avaliados os seguintes parâmetros da rede: indicador de intensidade do sinal recebido (Received Signal Strength Indicator - RSSI) e a relação sinal-ruído (Signal to Noise Ratio - SNR) no entorno do câmpus e em alguns pontos da cidade. Além disso, os dados obtidos foram confrontados com um modelo de cobertura da rede obtidos pela ferramenta CloudRF [CloudRF, 2019]. Neste trabalho também são apresentados o plano de expansão da rede e os pontos escolhidos para instalação das antenas e gateways. Algumas aplicações encontram-se na fase de desenvolvimento, como o rastreamento de veículos e o monitoramento da água e do ar. Tais aplicações são brevemente descritas ao término do artigo. Finalmente, os resultados demonstram a boa cobertura da rede e comprovam seu potencial para impulsionar soluções de cidade inteligente para o município e região.

Este artigo está organizado da seguinte forma. Na Seção 2 é apresentada uma breve comparação entre as principais tecnologias e padrões LPWAN, descrevendo o padrão LoRaWAN e os trabalhos relacionados. A Seção 3 descreve a implantação da rede, incluindo as escolhas tecnológicas e parâmetros de configuração. A Seção 4 detalha a metodologia de avaliação e os resultados obtidos. A Seção 5 apresenta o plano de expansão da rede e algumas aplicações atualmente em desenvolvimento. A conclusão e trabalhos futuros são apresentados na Seção 6. 


\section{Redes LPWAN}

A comunicação sem fio é um desafio, especialmente em aplicações de cidades inteligentes, visto que os objetos inteligentes têm sua capacidade de processamento limitada e devem operar com baixo consumo energético. Nesse contexto, destacam-se as redes de baixo consumo de energia e longo alcance denominadas LPWAN (Low Power Wide Area Network). As redes LPWAN permitem o envio de alguns Kbps a distâncias de até $50 \mathrm{~km}$ em campo aberto com um reduzido consumo de energia, proporcionando maior vida útil às baterias dos dispositivos [Raza et al., 2017]. Dentre as principais tecnologias LPWAN tem-se a Weightless SIG [Weightless SIG, 2019], NB-IoT (Narrowband Internet of Things) [Boisguene et al., 2017], SigFox [SigFox, 2019] e LoRaWAN [LoRa Alliance, 2017].

A rede NB-IoT baseia-se na rede licenciada LTE (Long Term Evolution), também conhecida como 4G, e já existente em operadoras de telefonia celular. A rede NB-IoT usa a topologia estrela com uma taxa de dados de $170 \mathrm{kbps}$ (downlink) e $250 \mathrm{kbps}$ (uplink), tendo como principais características o longo alcance (aproximadamente $35 \mathrm{~km}$ ) e o baixo consumo de energia. Além disso, os serviços de infraestrutura de rede são fornecidos por operadoras possibilitando a implantação rápida de sistemas para IoT, porém existe a cobrança pela utilização. No Brasil, a operadora TIM implantou em junho de 2018 uma rede piloto NB-IoT na cidade de Santa Rita do Sapucaí (MG) [Amaral, 2018]. Atuando na faixa de $700 \mathrm{MHz}$, usa um canal de $250 \mathrm{kHz}$ e também considera utilizar outras faixas como $1.800 \mathrm{MHz}, 2,5 \mathrm{GHz}$ e 2,1 GHz.

SigFox é uma tecnologia proprietária desenvolvida pela empresa francesa SigFox que atua como operadora de IoT. Nesse padrão o cliente adquire uma licença para uso da rede. SigFox compreende a camada de rede e de transporte e utiliza a tecnologia de banda ultra estreita (Ultra Narrow Band - UNB) que usa a largura do canal inferior a 1 $\mathrm{kHz}$ dentro da faixa ISM de $900 \mathrm{MHz}$ permitindo um alcance de $3 \mathrm{~km}$ a $10 \mathrm{~km}$ nas áreas urbanas e de $30 \mathrm{~km}$ a $50 \mathrm{~km}$ nas zonas rurais. Possui uma taxa de dados de $100 \mathrm{bps}$ transportando até 12 bytes de uplink, limitado ao envio de 140 mensagens/dia e 8 bytes de downlink com 8 mensagens/dia. Para utilização da SigFox é necessário fazer uso da rede da operadora e o pagamento de licença.

\subsection{LoRa e LoRaWAN}

A tecnologia de transmissão sem fio LoRa é desenvolvida e patenteada pela empresa Semtech. LoRa realiza a modulação de radiofrequência (RF) baseada no espalhamento de espectral de chirp (Chirp Spread Spectrum - CSS). Essa técnica é utilizada na comunicação militar e espacial permitindo cobrir longas distâncias de comunicação e alcançar robustez à interferência [LoRa Alliance, 2015]. Uma mensagem LoRa pode ser de uplink e downlink. A estrutura da mensagem é semelhante em ambos os casos, no entanto apenas a mensagem de uplink adiciona um código de verificação (CRC) que garante a integridade da carga útil (PHYPayload).

No Brasil, a faixa de utilização do LoRa é de $915 \mathrm{MHz}$, compreende a faixa de $902 \mathrm{MHz}$ a $907,5 \mathrm{MHz}$ e $915 \mathrm{MHz}$ a $928 \mathrm{MHz}$. Em agosto de 2018 a ANATEL publicou o Ato 6.506 que aprova os procedimentos para avaliação da conformidade de equipamentos de radiocomunicação de radiação restrita, permitindo a operação de dispositivos LoRa no território nacional. Apesar de ser possível utilizar dois padrões de transmissão, o 
americano e o australiano [LoRa Alliance, 2017], o último vem sendo adotado no Brasil. O padrão australiano possui 72 canais para uplink e 8 para downlink. Especifica que os canais de 0 a 63 utilizam uma largura de banda de $125 \mathrm{kHz}$ com uma taxa de codificação 4/5, com início em 915,2 MHz sendo incrementado linearmente em $200 \mathrm{kHz}$ até 927,8 MHz e os canais de 64 a 71 possuem uma largura de banda de $500 \mathrm{kHz}$ a partir de 915,9 MHz e incrementando linearmente de $1,6 \mathrm{MHz}$ a $927,1 \mathrm{MHz}$. E para downlink são os canais de 0 a 7 com uma largura de banda de $500 \mathrm{kHz}$, começando em 923,3 MHz e incrementando linearmente em $600 \mathrm{kHz}$ até $927,5 \mathrm{MHz}$.

Para usar a modulação LoRa é necessário configurar três parâmetros no dispositivo final. Essas configurações determinam o bit rate (quantidade de bits por segundo), o tamanho máximo do payload (carga útil de informações) e o air time (tempo em que o pacote está no ar), para permitir um maior alcance ou enviar uma quantidade maior de bits na mensagem:

- Largura de Banda (Bandwidth, BW): pode assumir três valores determinados, 125 $\mathrm{kHz}, 250 \mathrm{kHz}$ ou $500 \mathrm{kHz}$, sofrendo um deslocamento de até $20 \%$ que não influenciará na decodificação [Oliveira et al., 2018].

- Fator de Espalhamento (Spreading Factor, SF): determina o número de chirps necessários para representar um símbolo (um ou mais bits de dados), apresentando $2^{S F}$ valores possíveis de 7-12. Isso influencia a duração do um chirp: quanto maior o SF mais energia será usada por bit e maior será o alcance entre transmissor e receptor [Ortiz et al., 2018].

- Taxa de Código (Code Rate, CR): define o número de bits destinados para dados de redundância na mensagem, a fim de realizar a recuperação de erros, definidos entre 1 e 4 com os respectivos valores do CR: 4/5 , 4/6, 4/7 e 4/8. Incrementar o CR aumenta a proteção, mas também o tempo do bit no ar [Ortiz et al., 2018].

A Tabela 1 apresenta um exemplo de configuração de parâmetros dos dispositivos finais na faixa de $915 \mathrm{MHz}$ para dois cenários: maior alcance com payload máximo de 11 bytes de informação e maior mensagem com 242 bytes. Nota-se que quanto maior a distância, menor será o payload e maior será o tempo do pacote no ar, aumentando o consumo de energia pois o transmissor fica ligado por mais tempo.

Tabela 1. Exemplo de Configuração LoRa na Frequência de 915 MHz.

\begin{tabular}{lcc}
\hline Parâmetros & Maior Alcance & Maior Mensagem \\
\hline Data Rate (DR) & 0 & 4 \\
Spreading Factor (SF) & $\mathrm{SF} 10$ & $\mathrm{SF} 8$ \\
Bandwidth (BW) & $125 \mathrm{kHz}$ & $500 \mathrm{kHz}$ \\
Coding Rate (CR) & $4 / 5$ & $4 / 5$ \\
Bit Rate (BR) & $976 \mathrm{bps}$ & $12.500 \mathrm{bps}$ \\
Payload máximo & $11 \mathrm{bytes}$ & $242 \mathrm{bytes}$ \\
Air Time & $371 \mathrm{~ms}$ & $175 \mathrm{~ms}$ \\
\hline
\end{tabular}

LoRaWAN é um protocolo aberto de comunicação para a rede LoRa. Uma visão geral da arquitetura de uma rede LoRaWAN é mostrada na Figura 1. Os dispositivos finais (end nodes) são objetos com sensores ou atuadores. O gateway conecta os dispositivos na rede LoRaWAN com a Internet através do servidor de rede que gerencia a 


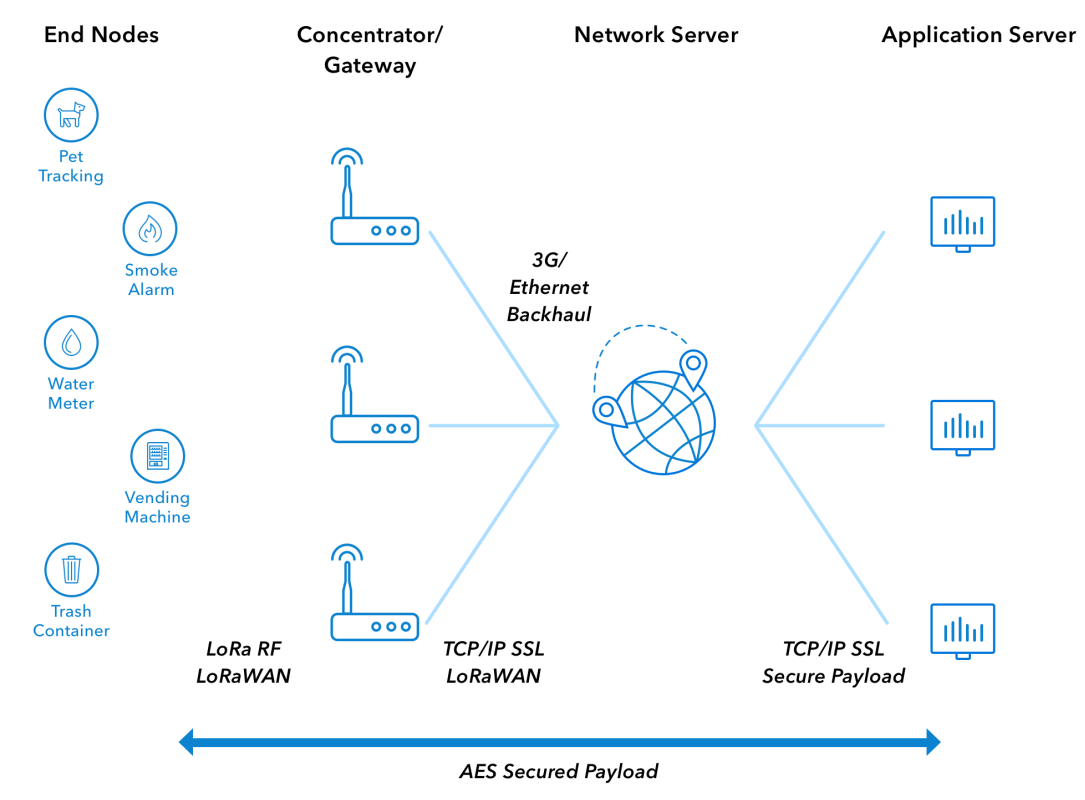

Figura 1. Visão Geral da Arquitetura LoRaWAN [LoRa Alliance, 2015].

comunicação dos dispositivos com o servidor de aplicação. O servidor de aplicação exibe as informações vindas dos dispositivos para o usuário final [LoRa Alliance, 2015].

Os dispositivo finais podem assumir três configurações. Classe A: configuração mandatória para as demais classes, nela o dispositivo final inicia comunicação com o gateway, realizando uma transmissão e abrindo duas janelas de recepção de dados do gateway. Classe B: a comunicação é iniciada pelo gateway, que determina o momento de transmissão do dispositivo final e na sequência abre duas janelas de recepção. Classe C: o dispositivo final realiza uma transmissão de dados, abre duas janelas de recepção e mantém uma aberta até a próxima transmissão, fazendo com que o dispositivo final necessite de uma fonte constante de energia.

LoRaWAN oferece através do padrão AES criptografia em dois níveis: o primeiro está localizado na camada de rede garantindo a autenticidade do dispositivo final e o segundo está na camada de aplicação e garante que a informação gerada no dispositivo somente será conhecida pelo servidor de aplicação na comunicação entre dispositivos finais e a aplicação. Há dois tipos de autenticação: OTAA (Over The Air Activation) e ABP (Activation By Personalization). A primeira é mais segura porque é o próprio dispositivo que gerencia sua ativação. No entanto, a autenticação de chaves via ABP é mais simples.

\subsection{Trabalhos Relacionados}

Diversos trabalhos apresentam aplicações de LoRaWAN no monitoramento da saúde [Mdhaffar et al., 2017], agricultura [Davcev et al., 2018; Carrillo \& Seki, 2017], monitoramento de dados ambientais [Addabbo et al., 2019], rastreamento de transporte público em tempo real [James \& Nair, 2017] e o impacto computacional da comunicação sem-fio Lora e da arquitetura de software do padrão LoRaWAN em plataformas de IoT na nuvem e na névoa [Zyrianoff et al., 2019]. Há também trabalhos que caracterizam e 
realizam análises teóricas e experimentais em solo europeu [Petäjäjärvi et al., 2015].

Uma caracterização da tecnologia LoRa em solo brasileiro é realizada em [Ortiz et al., 2018]. A partir do envio e recepção de mensagens realizado por um protótipo, os autores mediram a taxa de perda de pacotes, potência do sinal, relação sinal-ruído e de vazão. Essas medidas são obtidas variando o fator de espelhamento (SF) e a distância entre os nós do protótipo. Os autores concluíram que o melhor desempenho, considerando a taxa de transmissão teórica, consegue-se com o maior fator de espalhamento (SF11) pois oferece menor perda de pacotes. Em outro estudo, [Ortiz et al., 2019] apresentam uma análise comparativa entre os resultados de experimentos e simulações usando o software NS-3. Os resultados simulados a partir do modelo de propagação estocástico adaptado mostraram semelhanças com os experimentos reais, sendo equivalentes para todos os testes de desempenho usando os parâmetros de configuração da LoRa.

Uma revisão sistemática da literatura sobre aplicações de Lora e LoRaWAN foi realizada por [Oliveira et al., 2018]. A revisão reuniu 108 estudos e analisou 48 casos de aplicação. De acordo com os autores, há poucos estudos brasileiros sobre o padrão e o desenvolvimento da tecnologia LoRa em território nacional carece de avanços na área, principalmente implementações práticas em ambientes reais de monitoramento remoto.

\section{Implantação da Rede LoRaWAN}

A primeira etapa do trabalho consistiu na implantação da rede LoRaWAN na UTFPR - Câmpus Toledo, com a instalação física dos equipamentos que compõe o gateway no quarto andar do Bloco E, cerca de $20 \mathrm{~m}$ do solo. Também foi realizada a integração do gateway com o servidor de rede LoRaWAN e com o servidor de aplicação TagoIO. Tais componentes principais, mostrados no diagrama simplificado da Figura 2, são descritos a seguir:

- Gateway - foi utilizado um computador de placa única Raspberry Pi 2 juntamente com um concentrador LoRaWAN que possui o CI SX1301, fornecido pela empresa brasileira Radioenge e uma caixa hermética para armazenamento do gateway. Do lado externo do prédio foi instalada uma antena omni direcional de 6 dBi.

- Servidor de Rede LoRaWAN - existem diversos servidores para rede LoRaWAN, mas em sua maioria é cobrado por dispositivo ou por tráfego. Na implantação da rede foi utilizado o servidor da The Things Network (TTN) que é um projeto aberto, disponibilizado através de um servidor cloud e sem custos. No servidor TTN foram cadastrados o gateway, os dispositivos finais e a integração com o servidor de aplicação.

- Servidor de Aplicação - existem diversos servidores de aplicação como AllThingsTalk [AllThingsTalk, 2019], Cayenne [Cayenne, 2019], entre outros. Para esse trabalho foi utilizado o servidor TagoIO [TagoIO, 2019] como principal meio para armazenamento dos dados por oferecer uma solução cloud e uma plataforma gratuita para desenvolvedores de soluções para IoT. Conta com uma integração predefinida com o servidor da TTN que além de disponibilizar os dados no navegador Web também conta com aplicativos para Android e IOS. Também foi utilizado o servidor de aplicação TTN Mapper para coleta de informações.

O gateway faz uso da interface cabeada Raspberry Pi para enviar os dados para o servidor TTN. O acesso interno via SSH foi liberado para acessar o gateway. A instalação 


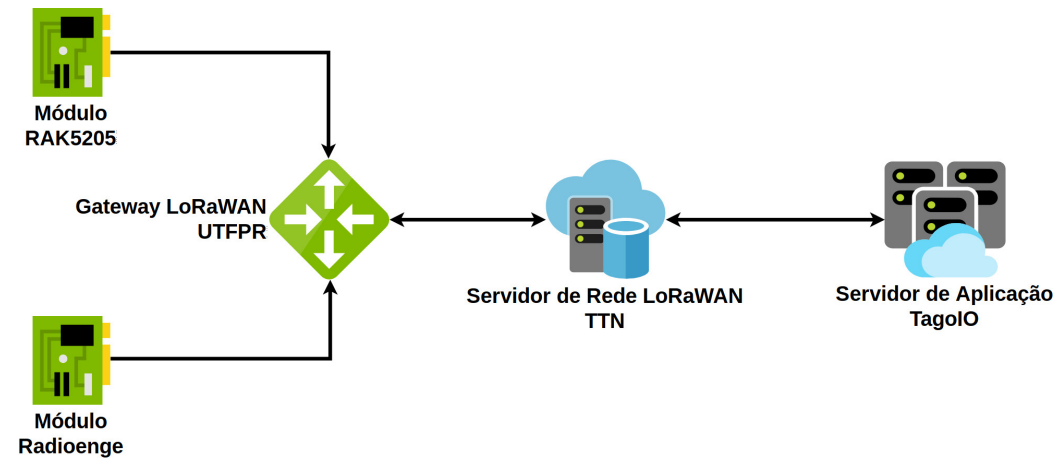

Figura 2. Diagrama da rede LoRaWAN.

ocorreu em maio de 2019 e vem se mostrando estável. Apenas 2 interrupções no funcionamento foram registradas: por interrupção na energia elétrica e por erro de operação.

\section{Avaliação da Rede LoRaWAN}

A partir da instalação física da rede utilizou-se a ferramenta Google Earth para estabelecer uma cenário de avaliação em torno do câmpus, mostrado na Figura 3. Foi definido um raio máximo de três quilômetros a partir do Bloco E, abrangendo uma área de aproximadamente $28,4 \mathrm{~km}^{2}$, elevação variando entre 428 e 567 metros em relação ao nível do mar e cobrindo tanto a zona urbana quanto rural do município. Também foram estabelecidas subáreas com um raio de 600 metros, sendo que foram mapeados os obstáculos que eventualmente possam impedir a comunicação entre o dispositivo final e o gateway.

Como não está disponível publicamente nenhum estudo sobre propagação de rádio frequência (RF) no espectro de $915 \mathrm{MHz}$ no município de Toledo utilizou-se a ferramenta CloudRF [CloudRF, 2019] para fazer a modelagem da propagação de RF na área de teste. CloudRF é um serviço online para modelar a propagação de radiofrequência na faixa de 20 a $100.000 \mathrm{MHz}$, oferecendo suporte a uma extensa lista de padrões específicos como AM, PWR, PTH, VHF/UHF, LoRa, GSM, UMTS, LTE, 5G, Wi-Fi, Wi-Max, Wi-Gig e outros. O serviço considera a física da propagação de sinais para estimar a cobertura e não a legislação imposta por órgãos reguladores. O CloudRF integra-se ao Google Earth. A perspectiva da pronação com base no relevo, não levando em consideração obstáculos como prédios, árvores e outros é mostrada na Figura 3b. Desta forma houve a necessidade de confrontar os dados simulados com uma avaliação in loco. Os trajetos são nomeados como "limitadores" L1-L8. Os limitadores L1-L5 e L8 representam um determinado caminho avaliado, já L6 e L7 são pontos únicos de avaliação. L1: sentido leste, compreende os pontos P1 a P11. L2: sentido norte, compreende os pontos P12 a P20. L3: sentido oeste, compreende os pontos P21 a P27. L4: compreende os pontos P28 a P33. L5: compreende os pontos P34-P36, juntamente com P28-P30, que fazem parte do trajeto. L6: apenas o ponto P37. L7: ponto P38 (Lago Municipal). L8: segue para oeste depois de L5 e compreende os pontos de longo alcance (pontos P39-P46) com distâncias maiores que $10 \mathrm{~km}$.

Conforme apresenta a Figura 3b, a CloudRF estabelece valores, associados a cores, que variam de $-5 \mathrm{dbm}$ até $-120 \mathrm{dbm}$. O valor de $-5 \mathrm{dBm}$ indica um sinal mais forte para a transmissão enquanto que $-120 \mathrm{dBm}$ indica o sinal mais fraco. As imagens geradas pelo CloudRF foram obtidas a partir da compra de créditos para estender o alcance para 


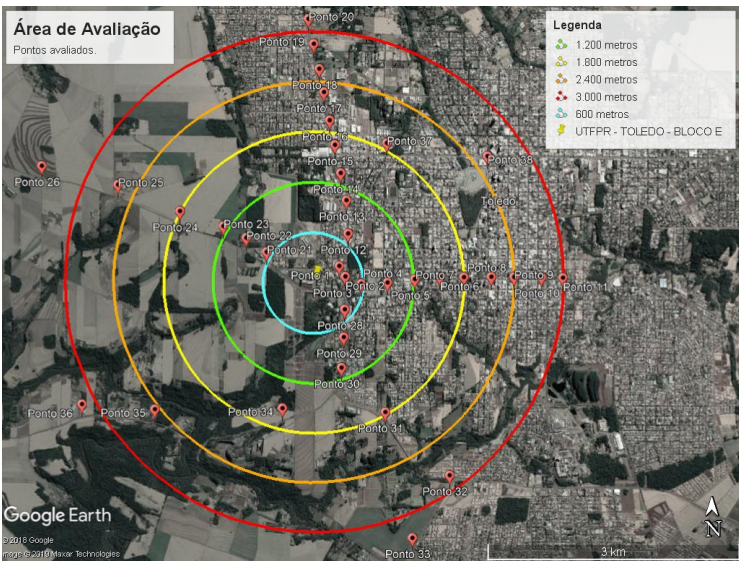

(a) Área de avaliação.

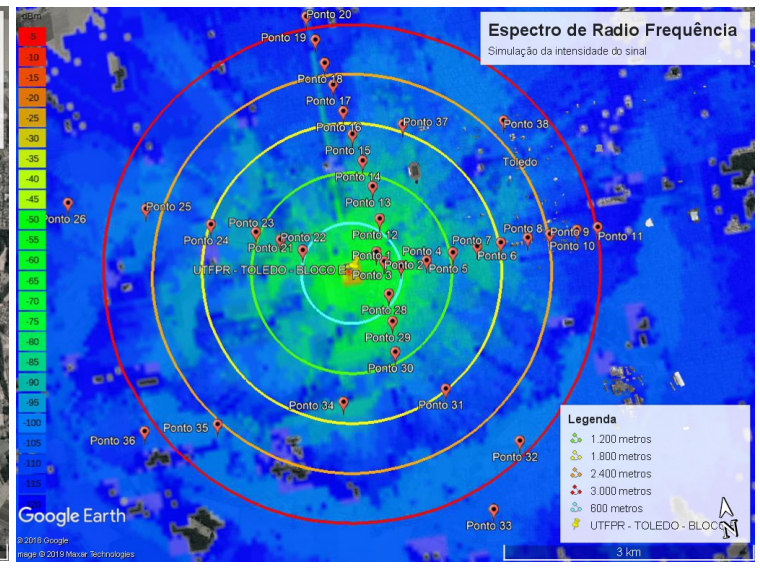

(b) Simulação através da CloudRF

Figura 3. Área de Avaliação.

$40 \mathrm{~km}$. A versão gratuita da CloudRF permite no máximo $10 \mathrm{~km}$.

Os dados que alimentaram o CloudRF são apresentados na Tabela 2. A configuração dos parâmetros no CloudRF iniciou com a especificação da frequência de operação que no caso desse estudo é de $915 \mathrm{MHz}$. Após isso, foi especificada a localização do gateway informando a latitude, a longitude e a altura da antena em relação ao solo (Height AGL). Para os dados da antena foram informados o azimute e o ganho da antena. Também foi necessário informar os dados do dispositivo final como a altura da antena do dispositivo em relação ao solo (Height(s) AGL) e o ganho da antena. O CloudRF traz vários modelos de propagação, para esse estudo foi utilizado o Irregular Terrain Model (ITM). Para exibição do modelo foi utilizada a resolução do terreno de $90 \mathrm{~m}$ com um raio de alcance de $40 \mathrm{Km}$.

Tabela 2. Parâmetros de Configuração CloudRF.

\begin{tabular}{lc}
\hline Parâmetros & Configuração \\
\hline Frequency & $915 \mathrm{MHz}$ \\
Latitude & -24.733594 \\
Longitude & -53.763812 \\
Height AGL & $20 \mathrm{~m}$ \\
Azimuth & $359^{\circ}$ \\
Antenna Gain & $6 \mathrm{dBi}$ \\
Height $($ s) AGL & $1,5 \mathrm{~m}$ \\
Gain & $5 \mathrm{dBi}$ \\
Propagation model & $\mathrm{ITM} /$ Longley-Rice $(<20 \mathrm{GHz})$ \\
Terrain resolution & $90 \mathrm{~m} / 198 \mathrm{ft}(\mathrm{DMS})$ \\
Radius & $40 \mathrm{Km}$ \\
\hline
\end{tabular}

\subsection{Metodologia de Avaliação}

Para aferir os pontos definidos na Figura 3a, um ponto de medição a cada 300 metros aproximadamente, empregou-se dois dispositivos finais que enviam periodicamente, ou por acionamento, a coordenada geográfica do local junto com uma mensagem LoRaWAN que indica o local avaliado, p.ex., "Ponto1", "Ponto2", etc. Ao receber a mensagem, 
o gateway calcula a intensidade do sinal e a relação sinal ruído da mensagem recebida. Em cada ponto da área de testes foram enviadas 5 mensagens. As áreas mais distantes tiveram o envio de 10 mensagens (pontos P39-P46). Todos os pontos foram registrados na ferramenta Google Earth. As informações geradas foram armazenadas no servidor de aplicação TagoIO indicando a comunicação dos dispositivos finais com o gateway e com os servidores de rede e de aplicação.

Dois dispositivos finais foram usados. O primeiro é uma placa WisTrio Rastreador RAK5205 desenvolvido pela empresa chinesa RAKwireless. A RAK5205 usa um CI LoRa SX1276 atuando na faixa de $915 \mathrm{Mhz}$ e um microcontrolador STM32L1, tem integrado um módulo GPS, além de outros sensores termômetro, barômetro, giroscópio, sensor de umidade e portas seriais. Já o segundo dispositivo é um módulo LoRaWAN desenvolvido pela empresa brasileira Radioenge. Ele é construído com um CI LoRa SX1276 e não conta com nenhum tipo de sensor, sendo apenas um módulo de comunicação. Diferentemente do RAK5205, o módulo Radioenge não possui um microcontrolador para o gerenciamento da comunicação. Assim para realizar o envio de mensagens foi acoplado um módulo base IOT-USB, também da Radioenge, adicionando uma interface USB que uma vez conectada ao computador permite o envio de mensagens para uma aplicação LoRaWAN.

Na primeira avaliação foi utilizado apenas o RAK5205 para envio das mensagens com as coordenadas GPS dos pontos de parada, porém a avaliação com esse dispositivo mostrou-se insatisfatória pois ele não possui comandos para configurar somente o envio das coordenadas, gerando um tráfego desnecessário com as leituras dos outros sensores. Além disso, o envio das coordenadas está atrelado a boa qualidade do sinal de GPS. Tal sinal sofre interferência direta em locais arborizados ou em períodos de céu nublado, fazendo com que o tempo de parada para realizar a avaliação de um ponto varie de 5 a 10 minutos. Diante disso foi necessário realizar uma nova avaliação modificando a maneira de obtenção dos dados.

Posteriormente, uma nova avaliação foi realizada com a utilização do módulo da Radioenge para o envio das mensagens e o RAK5205 apenas para complemento. Foi possível realizar medições em 46 pontos dentro e fora da área preestabelecida. O módulo Radioenge foi configurado com os parâmetros listados na Tabela 3, permitindo atingir o maior alcance e consequentemente um payload de apenas 11 bytes.

Tabela 3. Parâmetros de Configuração Radioenge.

\begin{tabular}{ccccc}
\hline Data Rate & Spreading Factor & Bandwidth & Coding Rate & Autenticação \\
\hline 0 & $\mathrm{SF} 10$ & $125 \mathrm{kHz}$ & $4 / 5$ & $\mathrm{ABP}$ \\
\hline
\end{tabular}

A adição do módulo da Radioenge na avaliação forçou a mudança do servidor de aplicação inicialmente escolhido, Cayenne, para o TagoIO [TagoIO, 2019]. Para enviar os dados para o servidor Cayenne é necessário estruturar a mensagem de uplink no dispositivo final, possibilitando que os dados sejam recebidos pela CayenneLPP e interpretados pela aplicação. Ao contrário da Cayenne, o TagoIO não necessita alterar o formato do payload enviado pelos dispositivos finais: ele recebe diretamente do handler o JSON com as informações, realizando o devido tratamento e exibição dos dados. 


\subsection{Resultados e Discussão}

Os resultados estão organizados em testes de curto alcance (área de teste) e de longo alcance (distâncias acima de $10 \mathrm{~km}$ ). Os resultados de curto alcance compreendem os limitadores L1-L7, enquanto o limitador L8 compreende os resultados de longo alcance.

A Figura 4 mostra um gráfico agrupando as aferições de curto alcance por limitador. Além da elevação do terreno, para cada ponto é exibido o melhor e o pior RSSI alcançado com o envio de cinco mensagens LoRaWAN. Para o SNR também são mostrados o melhor e pior SNR por ponto.

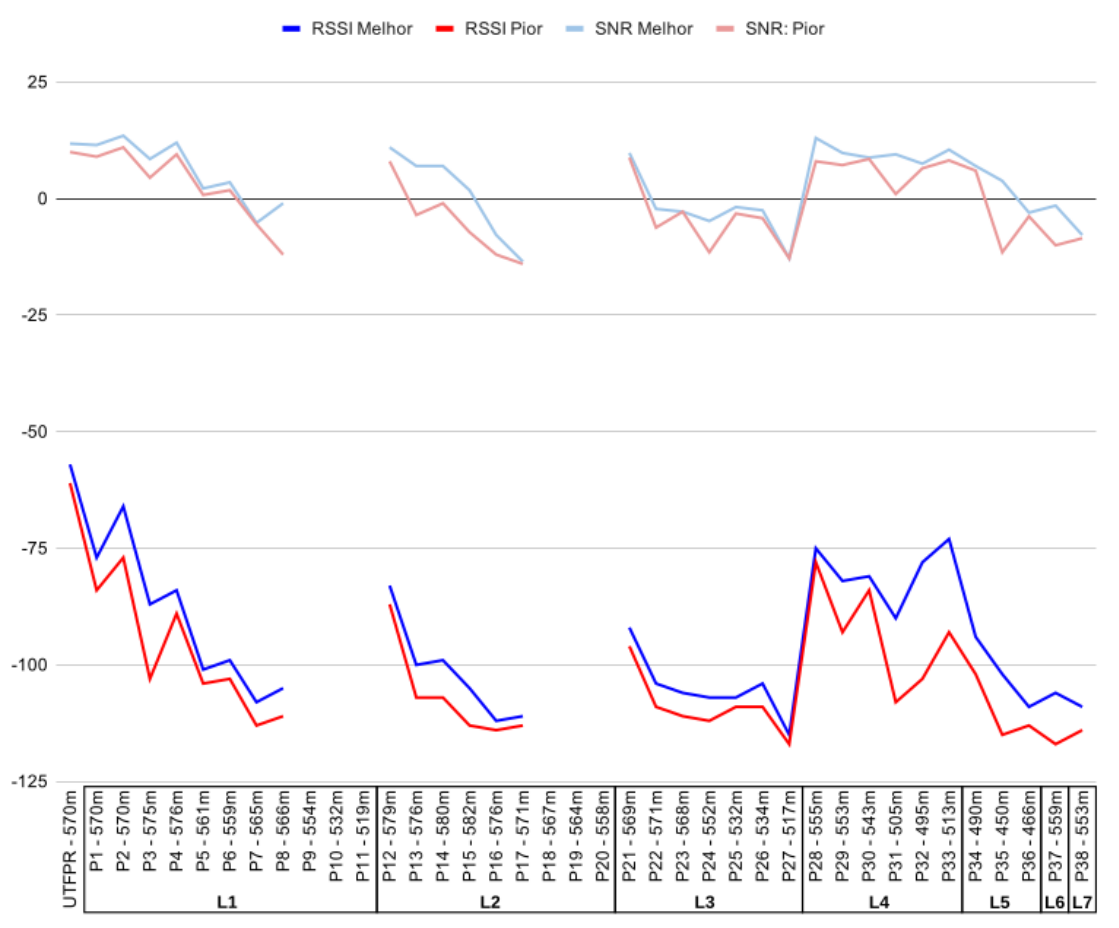

Figura 4. Resultados da avaliação da área de teste.

L1 engloba uma área urbana mais densa, indicando mais obstáculos (prédios, árvores e outros) que aumentam a degradação do sinal. Além disso, há um declive mais acentuado a partir do ponto P8 impedindo o envio de mensagem LoRaWAN nos pontos P9-P11, podendo-se notar a ausência de sinal em tais pontos na Figura 5. Cenário semelhante ocorre em L2, que apesar de estar na área urbana, não se tem um grande número de construções altas, porém apresenta uma elevação maior entre o gateway e os pontos P18-P20, impedindo o envio de mensagens LoRaWAN em tais pontos.

L3 concentra grande parte dos pontos da zona rural. Dessa maneira ele apresenta uma curva de degradação de sinal sem muitas oscilações como no L1 e apesar do SNR apresentar valores negativos não interferem no envio de mensagens LoRaWAN. L4 compreende uma área mista urbana e rural. Nota-se que não existe uma grande oscilação no SNR, porém há uma diferença maior entre o melhor e o pior RSSI, indicativo de uma possível interferência não identificada. L5 compreende maior parte na zona rural. A curva de degradação de sinal indica que houve uma diferença maior entre o melhor e o pior RSSI 


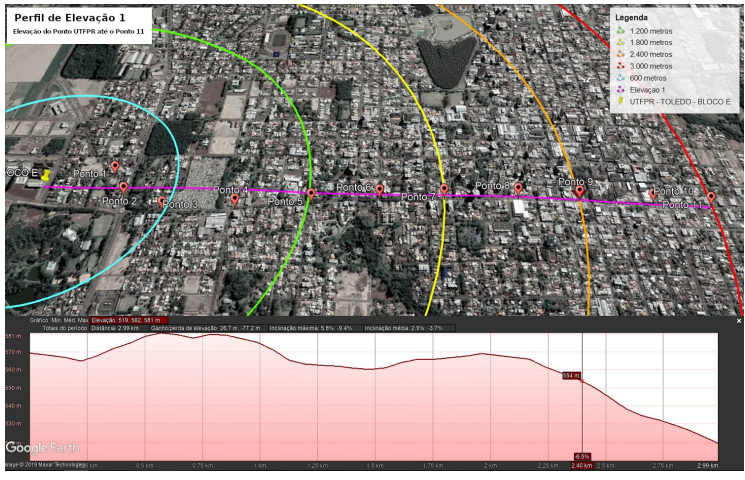

(a) Limitador L1.

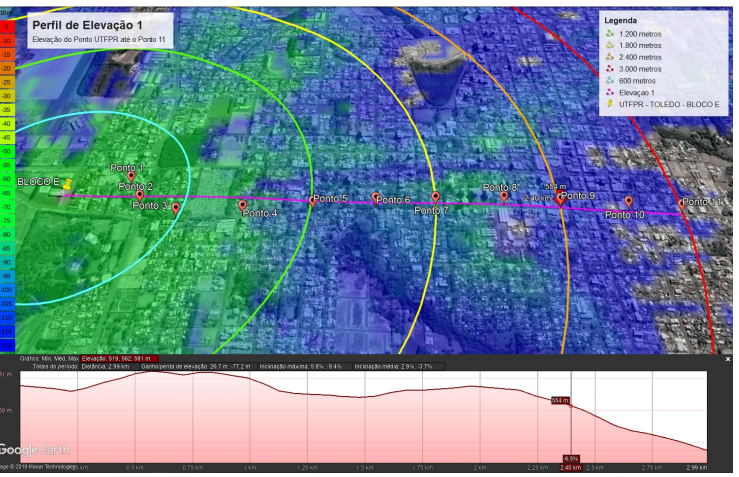

(b) Estimativa de L1 através da CloudRF

Figura 5. Elevação do limitador L1.

em virtude da grande quantidade de árvores presentes em P35. Já em relação ao SNR, quase não houve variação, exceto no $\mathrm{P} 35$, indicando que os obstáculos geraram ruídos no sinal, porém não interferiu no envio das mensagens LoRaWAN. Para complementar a avaliação na área de curto alcance, L6 estabelece a verificação diretamente do Ponto da UTFPR até o P37 e o L7 abrange o trajeto da UTFPR até P38. Essas avaliações foram realizadas apenas para verificar o relevo do terreno e o comportamento do sinal de RF. Esses limitadores atravessam o L2, isso indica que há um relevo mais alto entre o gateway e o dispositivo final, porém isso não impede a transmissão das mensagens LoRaWAN.

Na avaliação de longa, a distância L8 engloba os pontos P39-P46. A distância do Ponto da UTFPR para os pontos de parada variam de 12.296 até 32.098 metros e os resultados são apresentados na Figura 6. A curva de degradação do sinal não apresenta oscilações acentuadas, indicativo típico de uma zona rural. Também é possível visualizar que não há uma grande elevação do terreno entre o gateway e o último ponto avaliado. Apesar do SNR negativo e o RSSI muito baixo, não houve interrupção das mensagens LoRaWAN. Assim, os resultados apresentados mostraram que o relevo da região onde a rede LoRaWAN foi implantada influencia diretamente no envio das mensagens LoRaWAN. Obstáculos como prédios e árvores, por exemplo, prejudicaram a qualidade do sinal diminuindo o alcance de atuação do dispositivo final. Os resultados dos testes de longa distância demonstraram o potencial da rede LoRaWAN para áreas rurais mais afastadas. Apesar da grande distância entre o dispositivo final e o gateway, o RSSI e o SNR mantiveram-se constantes.

Finalmente, os resultados experimentais obtidos in loco corroboram a estimativa inicial de cobertura RF do modelo CloudRF. Assim, os resultados mostraram que a tecnologia LoRaWAN constitui uma alternativa de infraestrutura viável para o desenvolvimento de soluções para IoT, tanto para áreas urbanas quanto para rurais, a um custo relativamente baixo quando comparado com outras tecnologias.

\section{Expansão da Rede LoRaWAN e Aplicações}

A Figura 7 apresenta o plano de expansão da rede na área urbana da cidade em 2020. Ao total serão 6 pontos, considerando o ponto atualmente em operação na UTFPR. Os demais pontos foram escolhidos entre os imóveis do governo municipal que possuem infraestrutura de rede cabeada, estejam localizados em regiões elevadas da cidade e ofereçam estru- 


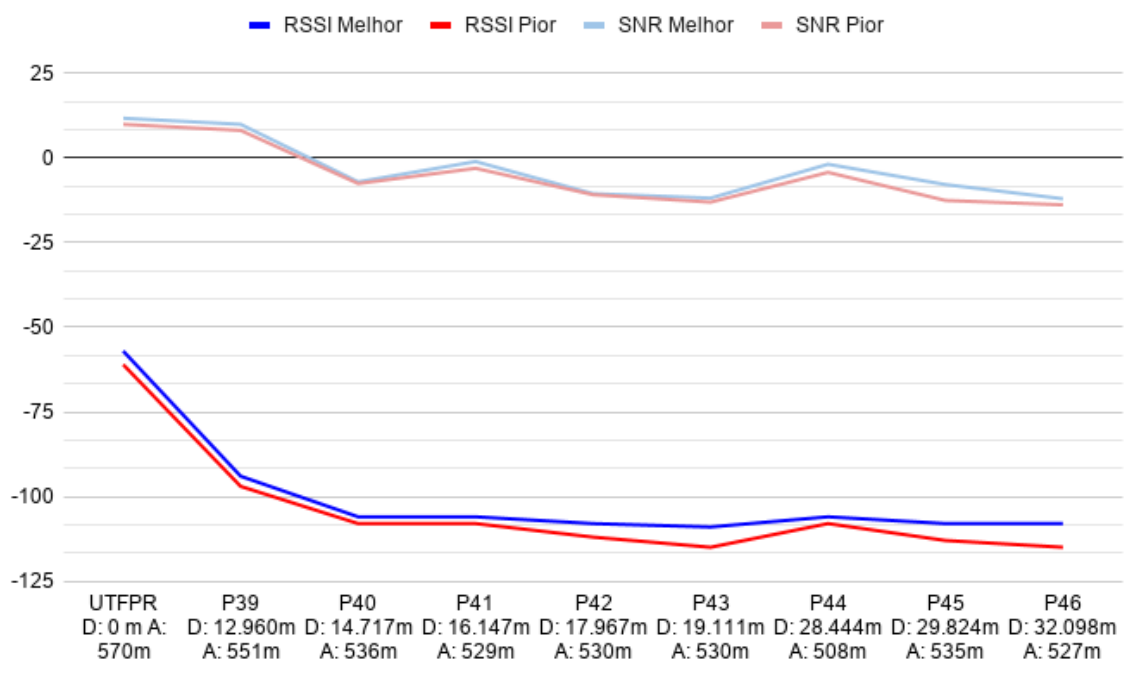

Figura 6. Resultados da avaliação de longo alcance.

tura física para instalar a antena em um ponto alto. Estima-se, considerando as avaliações obtidas na rede da UTFPR, que cada ponto terá uma cobertura mínima de $2,5 \mathrm{~km}$ de raio. É possível perceber que a região central da cidade contará com a intersecção de pelo menos 4 antenas.

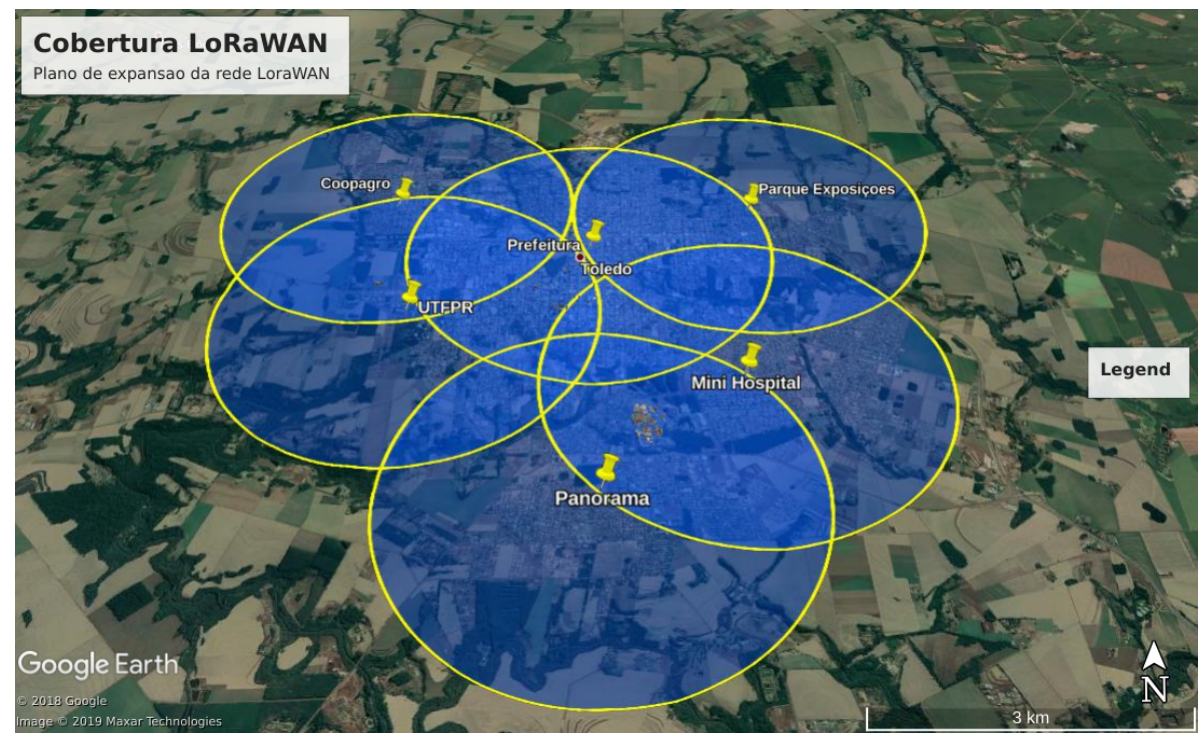

Figura 7. Plano de expansão da rede.

A colaboração entre a UTFPR e o Município de Toledo prevê o desenvolvimento de aplicações como rastreamento de veículos da coleta seletiva do lixo e monitoramento dos parâmetros da qualidade do ar, de acordo com a resolução 491 do Conselho Nacional do Meio Ambiente (CONAMA). Alguns testes com o protótipo de rastreamento de veículos estão em execução. Ao todo são dez caminhões que realizam a coleta seletiva de lixo no município e que devem receber o protótipo. Há também uma aplicação que acompanha em tempo real a umidade e temperatura em uma empresa localizada a cerca de $4 \mathrm{~km}$ da UTFPR. Sensores que coletam parâmetros da qualidade do ar serão incorporados a essa 
aplicação. Ambas aplicações enviam periodicamente os dados coletados. Para prototipar tais aplicações estão sendo empregadas placas de prototipação rápida (Arduino e ESP32), sensores diversos (geolocalização, umidade e temperatura), transmissores LoRaWAN, o servidor de rede TTN e o servidor de aplicação Tago. io.

\section{Considerações Finais}

Nesse artigo foi apresentada a implantação e avaliação de uma rede LoRaWAN na UTFPR - Câmpus Toledo como um passo importante para implementar o conceito de cidades inteligentes no município. Os resultados obtidos mostraram-se promissores e determinaram a cobertura alcançada para as zonas urbana e rural. Com apenas um gateway instalado na UTFPR foi possível cobrir quase que totalmente a zona urbana do município de Toledo. A zona rural também foi parcialmente contemplada pela cobertura da rede LoRaWAN, principalmente nas áreas mais próximas à UTFPR. Dessa maneira, os resultados experimentais demonstraram a viabilidade da utilização da tecnologia LoRaWAN como infraestrutura para o desenvolvimento de soluções para cidades inteligentes como o monitoramento de fluxo de veículos, transporte público, estacionamento rotativo, coleta de lixo, qualidade da água e do ar e outros. Apesar da propagação do sinal de RF ter sofrido influência direta da altitude do terreno, foi possível atingir uma distância superior a $30 \mathrm{~km}$, fazendo com que municípios vizinhos pudessem ser alcançados. De forma geral, concluise que a tecnologia LoRaWAN é viável para soluções de IoT principalmente quanto ao alcance de longas distâncias, seja em zonas urbanas e rurais. Assim, os resultados aqui apresentados servem como referência para novos estudos e desenvolvimento de soluções em IoT não só para o município de Toledo como também para região Oeste do Paraná.

\section{Referências}

Addabbo, T., Fort, A., Mugnaini, M., Parri, L., Pozzebon, A., \& Vignoli, V. (2019). Smart sensing in mobility: a lorawan architecture for pervasive environmental monitoring. In 2019 ieee rtsi.

AllThingsTalk. (2019). Allthingstalk - iot platform for inovative businesses and developers. Retrieved from https: / /www.allthingstalk.com/

Amaral, B. d. (2018). Tim lança piloto de nb-iot em $700 \mathrm{mhz}$ e projeta expansão para mais de mil cidades em 2018. Retrieved from https: / / inyurl.com/ teletimebr

Bardyn, J., Melly, T., Seller, O., \& Sornin, N. (2016, September). Iot: The era of lpwan is starting now. In Esscirc (p. 25-30). doi: 10.1109/ESSCIRC.2016.7598235

Boisguene, R., Tseng, S., Huang, C., \& Lin, P. (2017, June). A survey on nb-iot downlink scheduling: Issues and potential solutions. In 13th iwcmc (p. 547-551). doi: 10 .1109/IWCMC.2017.7986344

Carrillo, D., \& Seki, J. (2017, Aug). Rural area deployment of internet of things connectivity: Lte and lorawan case study. In Ieee intercon (p. 1-4). doi: 10.1109/ INTERCON.2017.8079711

Cayenne. (2019). Cayenne. Retrieved from https://tinyurl.com/cayenne -feat

Centenaro, M., Vangelista, L., Zanella, A., \& Zorzi, M. (2016, October). Longrange communications in unlicensed bands: the rising stars in the iot and smart city scenarios. IEEE Wireless Communications, 23(5), 60-67. doi: 10.1109/ MWC.2016.7721743 
CloudRF. (2019). Cloudrf web interface [Computer software manual]. Retrieved from https://cloudrf.com/

Davcev, D., Mitreski, K., Trajkovic, S., Nikolovski, V., \& Koteli, N. (2018, June). Iot agriculture system based on lorawan. In $W f c s$ (p. 1-4).

James, J. G., \& Nair, S. (2017, Nov). Efficient, real-time tracking of public transport, using lorawan and rf transceivers. In Tencon 2017 - 2017 ieee region 10 conference (p. 2258-2261). doi: 10.1109/TENCON.2017.8228237

LoRa Alliance. (2015). What is lorawan? [Computer software manual]. Retrieved from https://tinyurl.com/lorawan-def

LoRa Alliance. (2017). Lorawan 1.1 specification [Computer software manual]. Retrieved from https: / / inyurl.com/lorawan-spec

Mdhaffar, A., Chaari, T., Larbi, K., Jmaiel, M., \& Freisleben, B. (2017, July). Iot-based health monitoring via lorawan. In Ieee eurocon 2017 -17th international conference on smart technologies (p. 519-524). doi: 10.1109/EUROCON.2017.8011165

Oliveira, L. R., da Conceição, A. F., \& Neto, L. P. S. (2018). Revisão sistemática da literatura sobre aplicações das tecnologias lora e lorawan. In Viii brazilian symposium on computing systems engineering.

Ortiz, F. M., Almeida, T., Ferreira, A. E., \& Costa, L. H. (2019). Caracterização de desempenho de uma rede lora em ambientes urbanos: Simulação vs. prática. In Anais do iii workshop de computação urbana (pp. 167-180). SBC. doi: 10.5753/ courb.2019.7476

Ortiz, F. M., Cruz, P., de S. Couto, R., \& Costa, L. H. M. K. (2018). Caracterização de uma rede sem-fio de baixa potência e longo alcance para internet das coisas. In Anais do xxxvi simpósio brasileiro de redes de computadores e sistemas distribuídos. SBC.

Petäjäjärvi, J., Mikhaylov, K., Roivainen, A., Hanninen, T., \& Pettissalo, M. (2015, Dec). On the coverage of lpwans: range evaluation and channel attenuation model for lora technology. In 2015 14th international conference on its telecommunications (itst) (p. 55-59). doi: 10.1109/ITST.2015.7377400

Raza, U., Kulkarni, P., \& Sooriyabandara, M. (2017). Low power wide area networks: An overview. IEEE Com. Surveys Tutorials, 19(2), 855-873.

SigFox. (2019). Sigfox. Retrieved from https://www.sigfox.com

TagoIO. (2019). Tagoio. Retrieved from https://tago.io/en

Weightless SIG. (2019). Weightless. Retrieved from http://www.weightless . org/

Zyrianoff, I., Heideker, A., Silva, D., Kleinschmidt, J. H., \& Kamienski, C. (2019). Impacto de lorawan no desempenho de plataformas de iot baseadas em nuvem e névoa computacional. In Anais do xvii workshop em clouds e aplicações (pp. 4356). SBC. doi: 10.5753/wcga.2019.7593 